\title{
Characterization and differentiation potential of mesenchymal stem cells isolated from multiple canine adipose tissue sources
}

\author{
Usman Rashid', Arfan Yousaf ${ }^{1}$, Muhammad Yaqoob', Evelyn Saba², Muhammad Moaeen-ud-Din³, \\ Shahid Waseem ${ }^{4}$, Sandra K. Becker ${ }^{5}$, Gerhard Sponder ${ }^{5}$, Jörg R. Aschenbach ${ }^{5}$ and Mansur Abdullah Sandhu ${ }^{2 *}$
}

\begin{abstract}
Background: Mesenchymal stem cells (MSCs) are undifferentiated cells that can give rise to a mesoderm lineage. Adipose-derived MSCs are an easy and accessible source for MSCs isolation, although each source of MSC has its own advantages and disadvantages. Our study identifies a promising source for the isolation and differentiation of canines MSCs. For this purpose, adipose tissue from inguinal subcutaneous (SC), perirenal (PR), omental (OM), and infrapatellar fat pad (IPFP) was isolated and processed for MSCs isolation. In the third passage, MSCs proliferation/metabolism, surface markers expression, in vitro differentiation potential and quantitative reverse transcription PCR (CD73, CD90, CD105, PPARY, FabP4, FAS, SP7, Osteopontin, and Osteocalcin) were evaluated.

Results: Our results showed that MSCs derived from IPFP have a higher proliferation rate, while OM-derived MSCs have higher cell metabolism. In addition, MSCs from all adipose tissue sources showed positive expression of CD73 (NT5E), CD90 (THY1), CD105 (ENDOGLIN), and very low expression of CD45. The isolated canine MSCs were successfully differentiated into adipogenic and osteogenic lineages. The oil-red-O quantification and adipogenic gene expression (FAS, FabP4, and PPARY) were higher in OM-derived cells, followed by IPFP-MSCs. Similarly, in osteogenic differentiation, alkaline phosphatase activity and osteogenic gene (SP7 and Osteocalcin) expression were higher in OM-derived MSCs, while osteopontin expression was higher in PR-derived MSCs.
\end{abstract}

Conclusion: In summary, among all four adipose tissue sources, OM-derived MSCs have better differentiation potential toward adipo- and osteogenic lineages, followed by IPFP-MSCs. Interestingly, among all adipose tissue sources, MSCs derived from IPFP have the maximum proliferation potential. The characterization and differentiation potential of canine MSCs isolated from four different adipose tissue sources are useful to assess their potential for application in regenerative medicine.

Keywords: Mesenchymal stem cell, Adipose tissue, Dog, Adipogenesis, Osteogenesis

*Correspondence: mansoorsandhu@uaar.edu.pk

${ }^{2}$ Department of Veterinary Biomedical Sciences, Faculty of Veterinary

and Animal Sciences, PMAS-Arid Agriculture University, Rawalpindi 46300,

Pakistan

Full list of author information is available at the end of the article

\section{Background}

In the last decade, regenerative medicine has gained significant attention in human and veterinary medicine because of its potential role in healing or replacing apoptotic cells. The basic principle of regenerative medicine is to deliver stem cells to target sites that can promote the development of tissues/organs. A stem cells is an undifferentiated cell that is able to proliferate indefinitely and can differentiate into different types of cells. According 
to the source, stem cells are divided into embryonic stem cells, mesenchymal stem cells (MSCs) and induced pluripotent stem cells (iPSCs), as described by [1]. In canines, MSCs can be isolated from different parts of the body, including adipose tissue [2] bone marrow, umbilical cord [3], and synovial fluid [4]. International Society for Cell Therapy recommended three basic characteristics of the regenerative potential of MSCs, including plastic adhesion, the expression of clusters of differentiation (CD) including CD73, CD90, CD105, and multi-lineage differentiation [5]. Adipose-derived mesenchymal stem cells (AD-MSCs) have attracted attention because they can be easily collected by minimal invasive surgery. Contrasted with bone marrow-derived MSCs (BMSCs); AD-MSCs produce more cells, have a higher proliferation rate, and maintains their phenotype during higher passages [6]. Canine models are an excellent choice for studying various human musculoskeletal conditions because of their common pathological implications and clinical manifestation [7]. Past literature on canine species has proven the use of AD-MSCs for the treatment of osteoarthritis of the elbow [8], coxofemoral [9] and humeroradial joint [10].

In canines, inguinal subcutaneous adipose-derived MSCs showed a more rapid proliferation rate than omental adipose-derived MSCs; however, no differences were observed in lineage differentiation [11]. Another study on dogs showed that omental adipose tissue can be easily obtained regardless of the animal's condition, and always has a higher cell yield than subcutaneous adipose tissue [12]. The infrapatellar fat pad (IPFP) is another promising source of MSCs isolation and differentiation, with higher cell yield, colony-forming capacity, proliferation rate, and efficient differentiation to adipocytes as compared to subcutaneous adipose tissue and BM-MSCs [4]. However, most of the available studies in the literature on the isolation/differentiation of MSCs have compared two or three adipose tissue sources, or presents the comparisons between adipose tissue sources and bone marrow. The main objective of this study was to evaluate isolation, characterization, and differentiation efficiency of canine AD-MSCs due to their importance in veterinary medicine and their role as a study model for human musculoskeletal problems (Fig. 1).

\section{Results}

Isolation, and expansion of canine mesenchymal stem cells Stem cells were successfully isolated from SC, PR, OM, and IPFP cultured adipose tissue sources as shown in Fig. 2A-D. MSCs showed a typical fibroblast-like morphology, except for the MSCs recovered from IPFP,
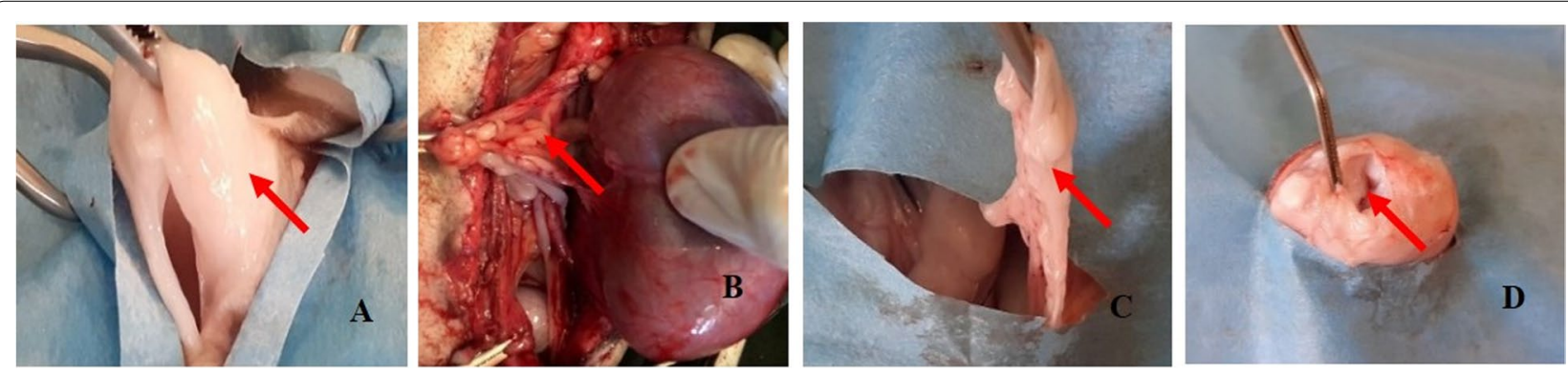

Fig. 1 Tissue collection sites of dogs. A. Inguinal subcutaneous, B. Perirenal, C. Omentum fat, D. Infrapatellar fat pad
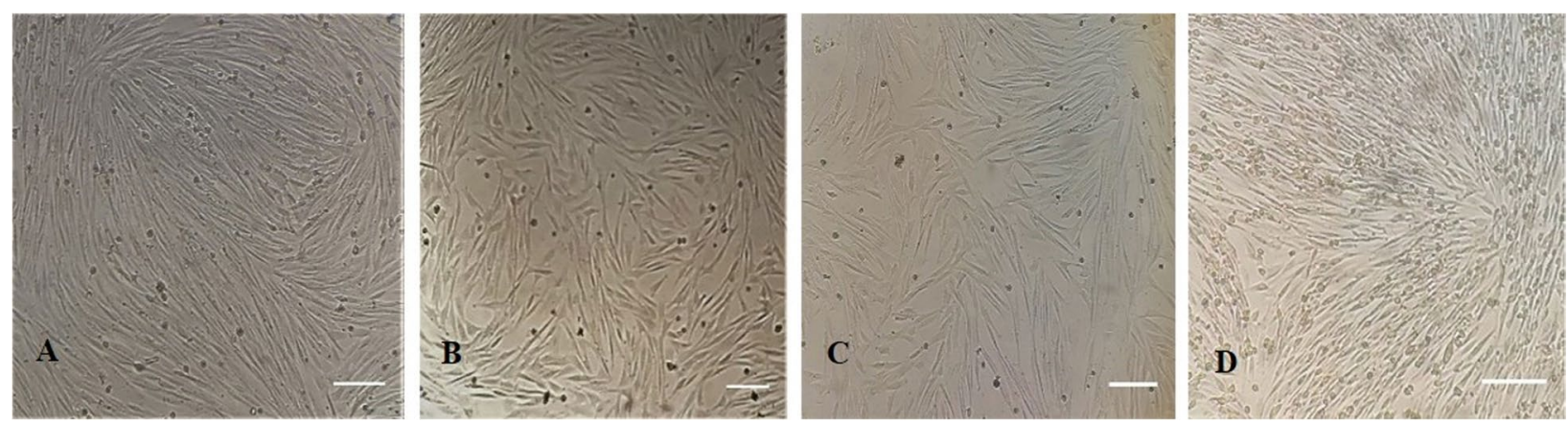

Fig. 2 The phenotype of canine MSCs at passage 3 (objective 20x). Panel A. Subcutaneous B. Perirenal C. Omental fat-derived MSCs. All recovered cells have a long spindle-shaped appearence, while, D. Infrapatellar fat pad derived MSCs were relatively smaller in size as compared to panel A, B, and C. Scale bar $=20 \mu \mathrm{m}$ 
the cell size was smaller than that of other AD-MSCs (Fig. 2D). However, all the separated MSCs adhere to the plastic surface, regardless of their source.

\section{Mesenchymal stem cells doubling time}

The cell doubling time of MSCs isolated from different sites was evaluated at P3 (Fig. 3A). On the 3rd and 6th day, cells from all sources showed non-significant $(P>0.05)$ change in the proliferation rate. However, the doubling rate of IPFP-derived MSCs increased significantly $(P<0.005)$ on the 9 th and 12 th days, while the proliferation rate of SC, PR, and OM-derived MSCs did not change significantly.

\section{Mesenchymal stem cells metabolic assay}

The metabolic activity of MSCs on days 3, 6, 9, and 12 was evaluated at P3. Our results showed that OM, SC, and PR derived MSCs have a significantly $(P=0.004)$ higher cellular metabolism compared to IPFP-MSCs. The metabolic activity of all adipose tissue-derived MSCs increased significantly $(P<0.003)$ on day 9 compared to that at day 3 . However, on day 12 th the cellular metabolic activity remained significant $(P<0.001)$ in comparison with all previous days irrespective of tissue origin (Fig. 3B).

\section{Immunophenotyping of mesenchymal stem cells}

At P3, the cell surface antigen expression of MSCs from all four fat sources was evaluated by flow cytometry and immunofluorescence. Our results revealed that cellular expression percentage of CD73 amounted to 56.2, 45.2, 42.9, 46.7\% in SC, PR, OM and IPFP, respectively, whereas the expression percentage of CD90 was 58.9,
53.2, 53.4, and 54.9\%, and the percentage of CD105 positive cells was $30.1,25.8,29.1$, and $27.6 \%$, respectively. The expression of CD45 in SC, PR, OM, and IPFP derived MSCs was 1.2, 1.74, 1.54 and $1.05 \%$ respectively, as shown in Fig. 4A. Adipose tissue-derived cells grown in complete LG-DMEM medium were evaluated for welldefined MSCs markers through immunophenotyping and conferred the scattered presence of CD73, CD90, and CD105 cell surface markers throughout the cell membrane, as shown in Fig. 4B.

\section{Adipogenesis}

To confirm adipogenesis, cells were stained with ORO for the identification of lipid droplets. The stained lipid droplets appeared red under in an inverted light microscope (objective $40 \times$ ) as shown in Fig. 5A. For quantitative analysis of ORO, the dye was eluted with anhydrous isopropanol and the absorbance was measured spectrophotometrically at $490 \mathrm{~nm}$. OM adipose-derived MSCs showed a significant difference $(P<0.001)$ in the concentration of ORO compared to undifferentiated precursor cells; however, SC, PR, and IPFP-derived MSCs did not develop significant differences in the concentrations of ORO during adipocyte differentiation as shown in Fig. 5B. The differentiated adipocytes showed the presence of FABP4 on the cell membrane and around the accumulating cytoplasmic lipid vacuoles (Fig. 5C).

\section{Osteogenesis and alkaline phosphatase activity (ALP)}

The osteogenic abilities of MSCs isolated from different sites were evaluated by treating them with osteogenic growth media for a period of 21 days and staining with Alizarin Red Stain. The differentiated cells showed

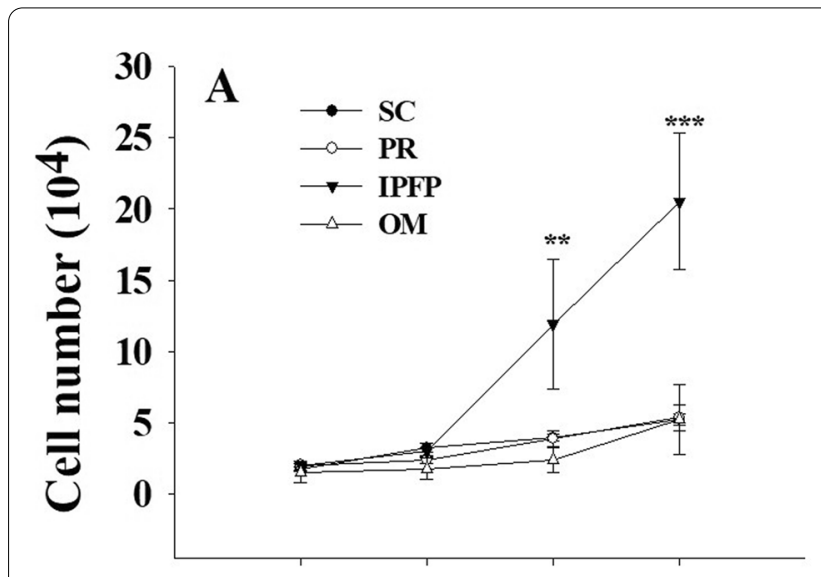

Day 3 Day 6 Day 9 Day 12

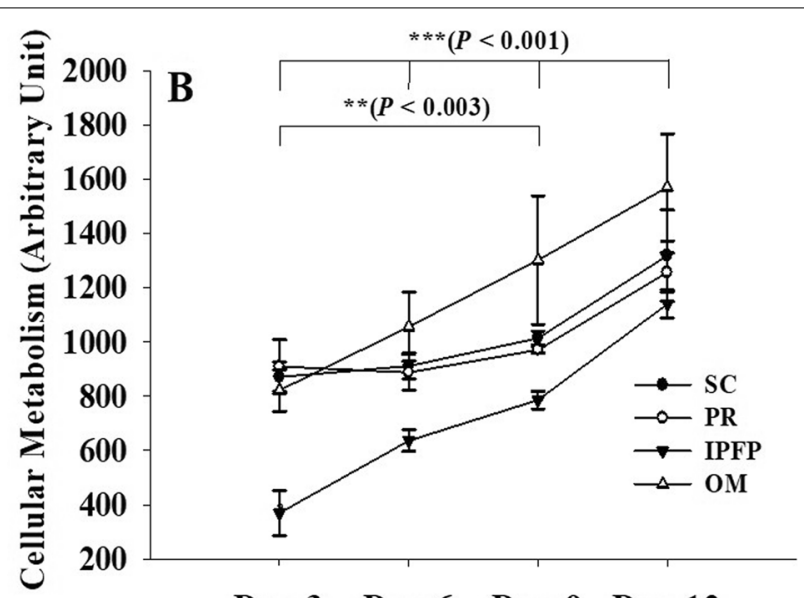

Day 3 Day 6 Day 9 Day 12

Fig. 3 (A) Cell doubling time on days 3,6,9, and 12. ${ }^{* * *} P<0.001,{ }^{* *} P=0.004$. (B) Cellular Metabolism (MTT assay) at day 3, 6, 9 and 12. The data is shown as mean \pm SEM. Significance among days, ${ }^{* *} P<0.001,{ }^{* *} P=0.003$. Abbreviations: S/C: Subcutaneous, PR: Perirenal, OM: Omentum, IPFP: Infrapatellar fat pad 


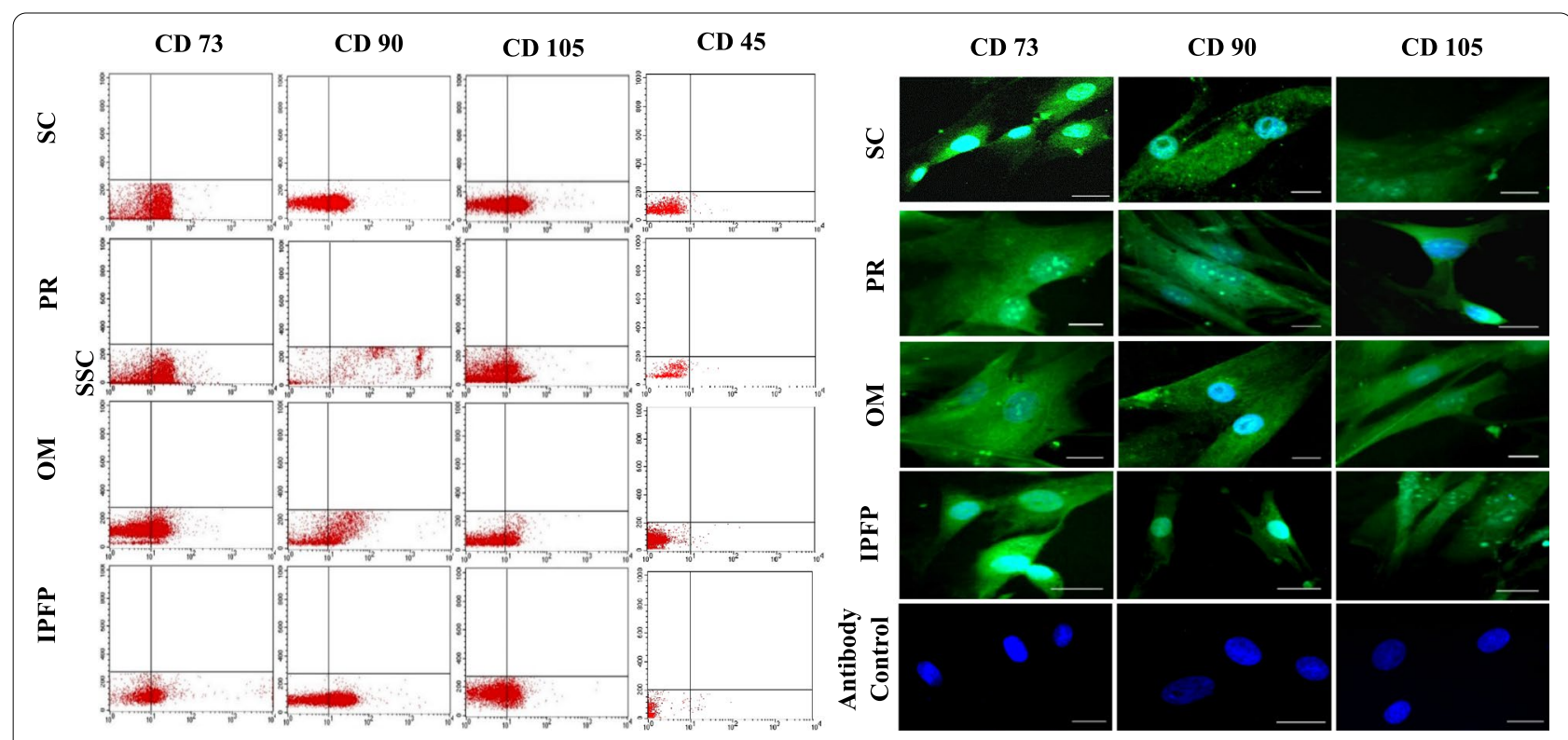

Fig. 4 (A) Flow cytometric expression of CD73, CD90, CD105, and CD45 in undifferentiated canine MSCs, derived from different fat sources. (B) Immunostaining of undifferentiated cMSCs derived from different tissue sources. The cells were stained positive with surface antigens CD73, CD90, and CD105 (green) and nuclear staining (DAPI, blue). Scale bar =25 $\mu$ m. Abbreviations: S/C: Subcutaneous, PR: Perirenal, OM: Omentum, IPFP: Infrapatellar fat pad

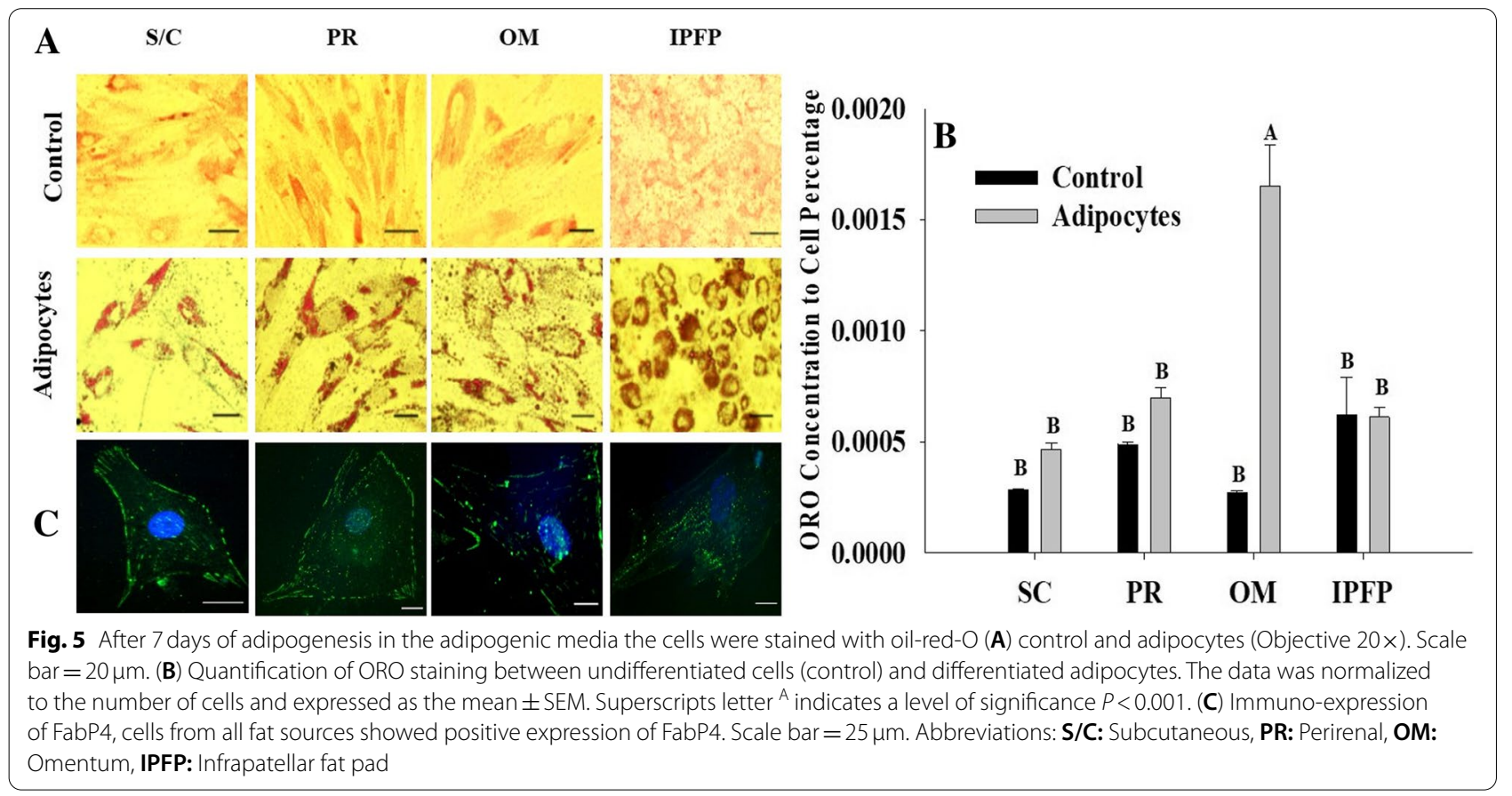

positive staining of the extracellular mineral matrix, as shown in Fig. 6A. Alkaline phosphatase activity is considered to be an early sign of osteogenesis. Compared with undifferentiated cells, the ALP activity of osteo-induced canine MSCs was significantly higher $(P<0.01)$. Furthermore, as shown in Fig. 6B, the ALP activity of differentiated cells from $\mathrm{OM}$ was higher $(P \leq 0.05)$ than that of cells from SC, PR, and IPFP. Osteocytes differentiated 


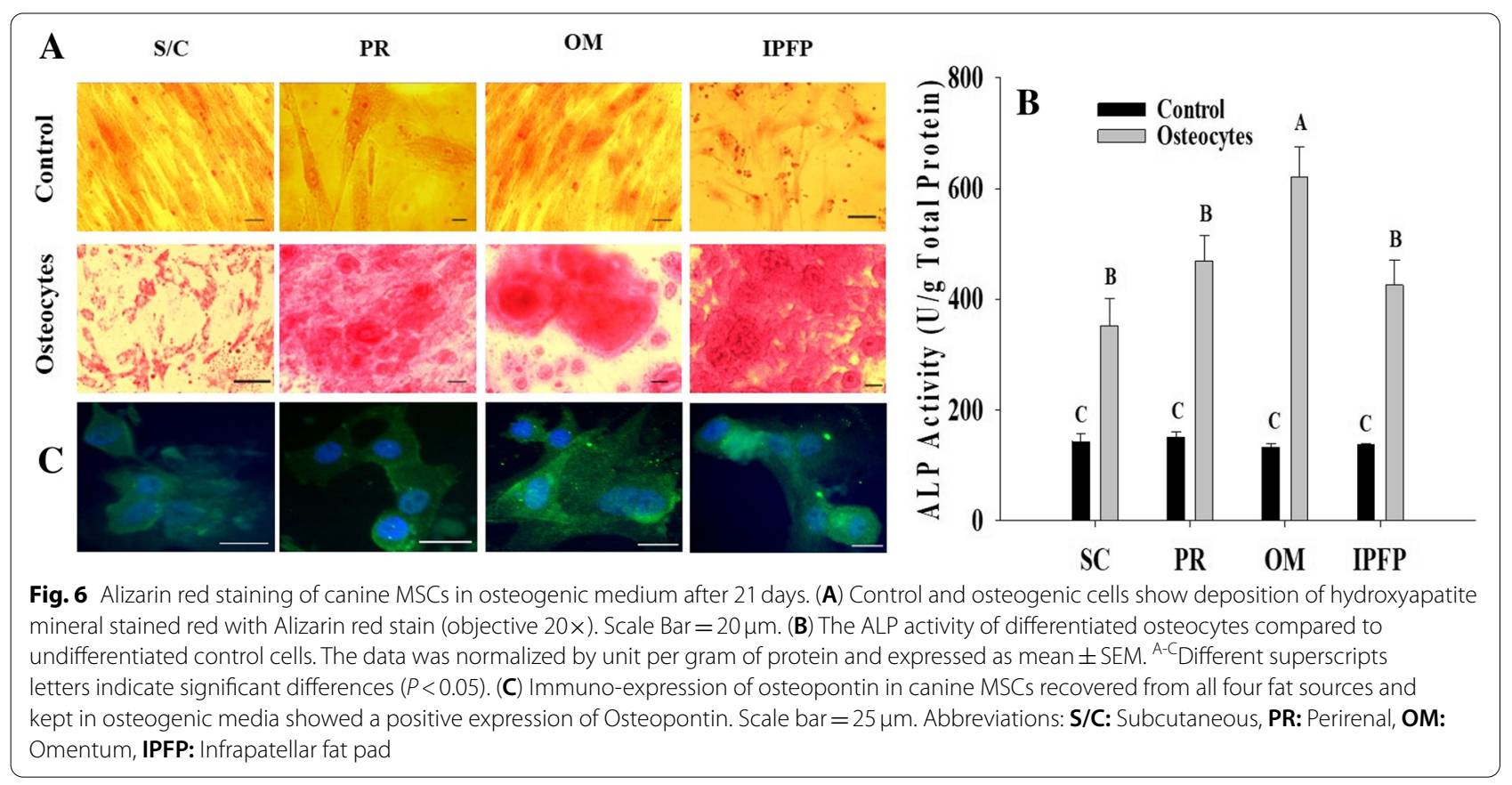

from all MSC sources showed positive expression of osteopontin as shown in Fig. $6 \mathrm{C}$, though the antigen was diffusely distributed all over the cells.

\section{Gene expression studies}

The gene expression of $C D 73, C D 90$, and $C D 105$ was tested in undifferentiated canine MSCs. Our results indicated that regardless of the tissue source the expression of $C D 73(P=0.577), C D 90(P=0.113)$ and $C D 105$ $(P=0.406)$ were non-significant as shown in Fig. 7A-C. Therefore, the results of qPCR confirmed that the cells used in this study were canine MSCs. Further, the expression of adipogenic markers (FAS, FABP4, and PPAR $\gamma$ ) revealed that, among all fat sources, OM-derived MSCs had significantly $(P<0.01)$ higher expression of $F A S$. In contrast, regardless of the tissue source expression of FABP4 $(P=0.386)$ and PPARY $(P=0.418)$ did not change significantly during the differentiation process as presented in Fig. 7D-F. However, compared to control cells, adipocytes differentiation from OM and IPFP fat sources tended to show a higher expression of FABP4 and PPAR . When MSCs were differentiated to osteogenic lineage, they were evaluated for osteogenic markers (SP7, osteocalcin, and osteopontin) as shown in Fig. 7G-I. Our results showed that, compared with the other four sources, the expression of SP7 in OM fat-derived MSCs was relatively higher, but the interaction of $S P 7 \times$ tissue sources was still non-significant $(P=0.627)$. Correspondingly, compared with MSCs recovered from PR and IPFP, the expression of osteocalcin in OM and SC adipose-derived
MSCs was higher, while the osteocalcin $\times$ tissue sources interaction remained non-significant $(P=0.951)$. Compared with all other tissue sources, the expression of osteopontin was higher in PR-derived MSCs as, but the osteopontin $\times$ tissue sources interaction remained nonsignificant $(P=0.995)$.

\section{Discussion}

Various studies have reported isolation of canine MSCs from the adipose tissue, umbilical cord blood, Wharton's jelly, and bone marrow [2, 3]. Adipose tissue is the most easily and readily available source for adult MSCs isolation. Most studies on the isolation of canine MSCs from adipose tissue have compared two or three different sources of adipose tissue $[12,13]$. In our study, canine MSCs were isolated from four different adipose tissue sources (including SC, PR, OM, and IPFP) of the same animal. The cells from all four adipose sources fulfilled the criteria of MSCs [5]. The MSCs released after enzymatic digestion of adipose tissues from different origin exhibited typical fibroblast morphology, similar to those of AD-MSCs obtained from humans [14] and dogs [11]. IPFP-derived cells have a fibroblast-like morphology, but are relatively small, as previously reported by [4]. The proliferation potential among SC, PR, and OM-derived MSCs remained non-significant in our study and similar results have been reported in humans [14] and dogs [12]. However, the proliferation rate of IPFP-derived cells was significantly higher, which may be due to their smaller cell size $[4,13]$. Vice versa, MTT assay demonstrated that 

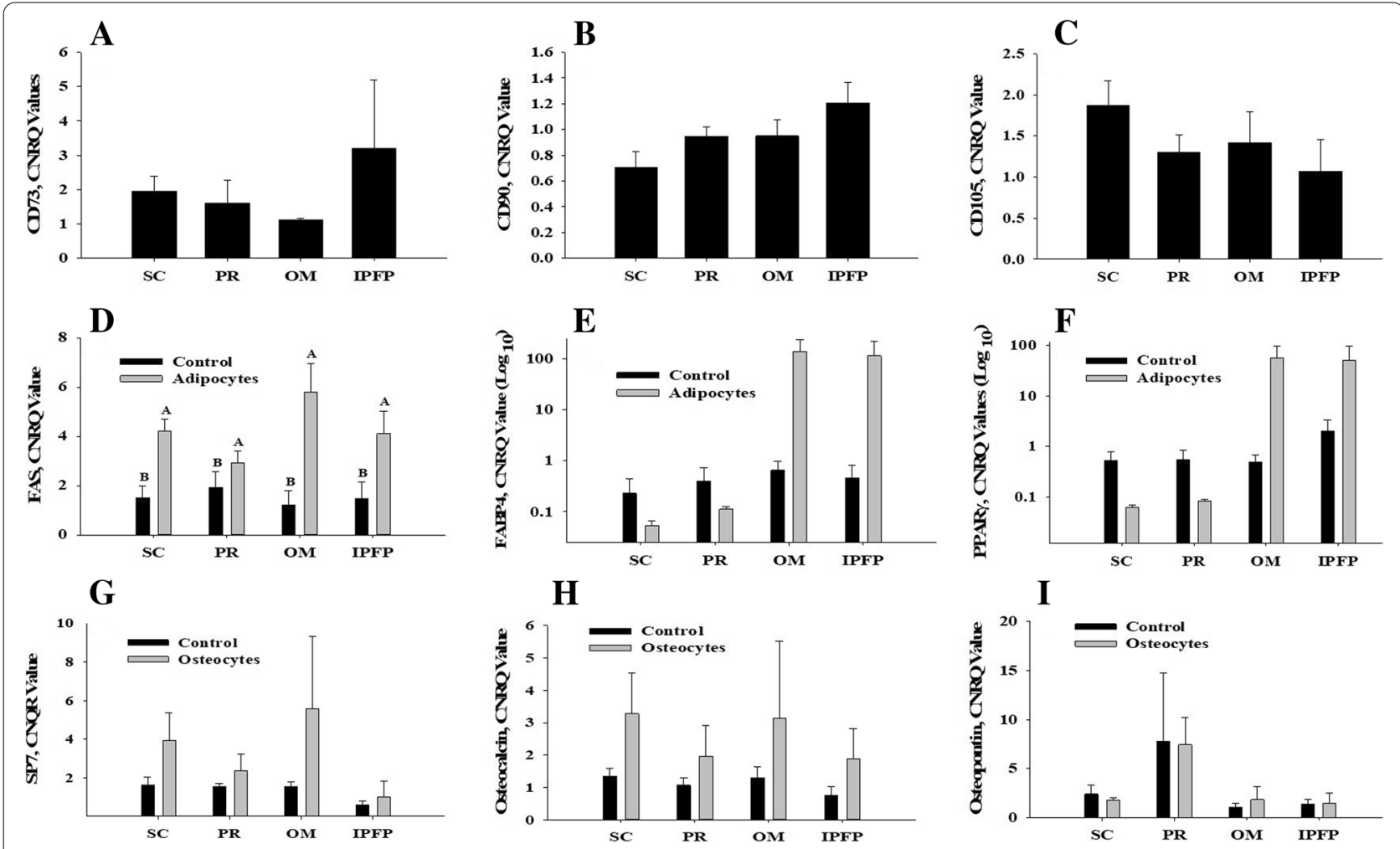

Fig. 7 At P3, the MSCs from all four fat sources were evaluated for the relative mRNA expression (CNRQ) of CD73 (A), CD90 (B), and CD105 (C) genes. After 7 days of adipogenesis, the differentiated adipocytes were evaluated for expression of FAS (D), FABP4 (E), and PPARY (F). While, the expression of osteogenic genes SP7 (G), Osteocalcin (H), Osteopontin (I) were evaluated in differentiated cMSC after 21 days in osteogenic media. The data was presented as mean \pm SEM. ${ }^{A, B}$ Different superscripts letter indicates significant differences within a given adipocyte source $(P<0.001)$. Abbreviations: S/C: Subcutaneous, PR: Perirenal, OM: Omentum, IPFP: Infrapatellar fat pad

the SC, PR, and OM-derived MSCs had higher cellular metabolism as compared to small-sized IPFP-MSCs. This response is directly proportional to the content of cell mitochondria and cell size [15]. On the 12th day of cellular propagation, the increase in IPFP-derived MSCs was associated with high mitochondrial biogenesis, which increased the number of cells [16]. All adipose-derived MSCs showed a positive expression of CD73, CD90, and CD105, which is in line with studies on MSCs isolated from canine [2, 4], bovine [17] and human adipose tissue [18]. In bovines, CD73 was more expressed in the Golgi apparatus and less on the cell membrane [17], but in our research, the expression of CD73 on cell membrane clearly indicates the difference in cell stage. CD73 acts as a signal and adhesion molecule to support cells anchoring in vivo and in vitro [19]. The expression of CD73 in $\mathrm{AD}-\mathrm{MSCs}$ from all four tissue sources was further confirmed by flow cytometric and qPCR analysis, and our results are in line with previously reported studies in dogs [20]. Another study on canines reported reduced expression of CD73 [2] which is not in agreement with our study, maybe due to the sample collection method or different anatomical sites of tissue. CD90 is another important marker of MSCs, and plays a vital role in cell adhesion, migration, and cell-to-cell/-matrix interaction [21]. As previously reported in humans, CD90 is strongly expressed in all tissue-derived cells [22] at the same time, some studies in mice and dogs have reported abridged expression of CD90 [23, 24]. Therefore, the positive expression of CD90 on MSCs depends on the species, tissue source, passage number and culture conditions $[4,17$, 23]. In this study, the expression of CD105 was relatively low compared with CD73 and CD90. While the expression of CD105 depends on the passage number and the site of tissue collection [20]. In murine, AD-MSCs with low CD105 expression may develop in the direction of adipocyte and osteocyte lineages [25]. CD45 is a cell surface tyrosine phosphatase that is present on hematopoietic origin cells containing the nucleus, including hematopoietic stem cells, but not on the non-hematopoietic origin cells. Therefore, CD45 is believed to be an elite marker of the hematopoietic lineage [2, 23]. Coherent with this fact, our study reported a very low expression of CD45 as compared to CD73, CD90, and CD105. 
In comparison to human MSCs, no standard criteria have been developed for the characterization of canine MSCs [26]. Such criteria are helpful because some contamination of culture with non-MSCs are inevitable during culture. Contamination with other cell types can be avoided or at least restricted by enhancing the culture density or repetitive media change and will decrease during passaging under optimized culture conditions [27]. It needs to be considered that the cells used in the present study represented a very early passage (P3). Nonetheless, these MSCs effectively differentiated into adipocytes with abundant cytoplasmic lipid droplets. Previous studies reported unsuccessful adipogenic differentiation of canine MSCs using human media [11], but our research method has efficiently differentiated canine MSCs into adipocytes. Compared with IPFP, the increase in ORO accumulation in OM-derived MSCs may be due to the increase in cell size. Apart from ORO concentration, the increased expression of PPAR, FABP4 and FAS genes also supports the MSCs differentiation into adipocytes.

The modified concentrations of dexamethasone, rosiglitazone, IBMX, and insulin worked very well in our experiments and have effectively differentiated MSCs into adipocytes within 7 days' time. In contrast, [28] reported the loss of adipogenic differentiation due to the presence of IBMX in canine induction media. Our results are consistent with those of Ritter et al. [29] who pointed out that the adipogenesis of human-derived OM was higher than that of SC cells. Another study did not describe any difference in adipogenesis between $\mathrm{OM}$ and $\mathrm{SC}$ derived AD-MSCs [2], this response may be due to the use of low glucose medium in the current study. However, when we cultured MSCs in high glucose medium, our cells developed to the senescence stage (data not shown). Dexamethasone is used in both adipogenic and osteogenic differentiation of MSCs and in combination with IBMX, it can promote PPAR $\gamma$-induced adipogenesis [30]. Rosiglitazone is also a PPAR $\gamma$ agonist, which accelerates the sensitivity of cells to insulin and promotes conversion into adipocytes. The adipogenic differentiation also requires insulin to initiate the transport of glucose, cholesterol, and fatty acids to the developing adipocytes. That's why PPARY is considered an early and FABP4 as a late marker of adipogenesis based on FABP4 involvement in the cellular transportation and metabolism of fatty acid [31]. An increased expression of PPARy and FABP4 mRNA was observed in OM and IPFP-originated cells, while the inconsistent qPCR results of SC and PRderived MSCs may be due to the shortened time (7 days) provided in the differentiation medium. Even though the change remained non-significant, this difference may be due to the increased adipogenic potential of $\mathrm{OM}$ and IPFP-derived cells, or the co-incubation of cells with
IBMX/rosiglitazone. The increase in FAS mRNA expression in differentiated cells from all sources indicates that the cells have a high demand for fatty acids and seems to be related to the potentiated action of insulin during adipocytes development [32].

Canine MSCs from all four sources were effectively differentiated into osteocytes and stained positively with ARS. During the process of osteogenesis, cells regularly detach from the surface and this phenomenon has already been reported by [4]. However, we did not observe any detachment in IPFP-derived cells during the osteogenesis process. Cells isolated from OM adipose tissue showed significantly higher ALP development, because differentiated cells express RUNT-related transcription factors that regulate osteogenic genes, including $A L P$ and osteocalcin [33]. One of the early osteoblast differentiation factors is ALP [34]. Regardless of our tissue source, ALP was high in all differentiated cells. In our experiment, the gene expression of osteopontin (second early osteogenic marker) was non-significant to that of the control group. This response may be because we checked the expression 21 days after osteogenesis when gene expression of osteopontin may have been reduced [35]. Even though, the immunocytochemical positive expression of osteopontin indicates that the antigen exists outside the cell and plays an important role in the accumulation of hydroxyapatite minerals. Osterix $/ S P 7$, a zinc finger transcription factor, plays a key role in the differentiation of MSCs into osteoblasts [36]. Compared with control samples, osteogenic differentiated cells from all adipose tissue sources (SC, PR, OM, and IPFP) showed high levels of SP7 and osteocalcin. The osteogenic differentiation induced by dexamethasone may increase the expression of osteocalcin through the formation of hydroxyapatite minerals in the presence of $\beta$-glycerophosphate [37]. In the process of mineralization, the importance of dexamethasone concentration for the gene activation of osteocalcin and osteogenic differentiation has also been reported [38]. Our findings indicated that, among all four sources, the OM and IPFP-derived MSCs have the better bi-lineage differentiation ability. On the other hand, IPFP derived MSCs have the highest proliferation rate among the studied sources of MSCs. Previous studies on canine reported the successful treatment of osteoarthritis using AD-MSCs and platelet-rich plasma (PRP) [39]. Similarly, the use of IPFP derived MSCs along with autologous PRP gels have shown promising results in the treatment of canine bone defects [40]. These studies provide solid evidence for the clinical application of AD-MSCs in canines. Many other factors including age, gender, cell isolation and differentiation protocols also affect the biological characteristics of stem cells [41]. A study on canine AD-MSCs reported an inverse correlation between cryopreservation and 
proliferation ability, but did not have a negative impact on the multilineage differentiation ability. In cell-based therapies, cell senescence is another important factor that MSCs show in higher passages. In higher passages, cells may become biologically exhausted, leading to poor proliferation and differentiation abilities [42]. Vice versa, another study conducted in our laboratory showed that freshly harvested autologous canine IPFP-MSCs showed significant proliferation rate and differentiation capacity, and they were successfully transplanted into critical sized bone defects [40,43]. Overall, our present study provides a novel comparison for the isolation, characterization, and differentiation of canine AD-MSCs from different anatomical origins of the same animal.

\section{Conclusions}

In conclusion, functional and molecular evidences suggest that canine $\mathrm{OM}$ adipose tissue is an excellent source for MSCs isolation and lineage differentiation. In addition, IPFP-based MSCs also have enhanced proliferation capacity and comparable adipogenic or osteogenic competencies. Our results suggest that, tissue source and proliferative potential should be considered an important aspect of stem cell therapy along with lineage differentiation abilities.

\section{Methods}

\section{Tissue collection}

Three severely injured dogs (6-10 months) were presented at the outdoor clinic of the Faculty of Veterinary and Animal Science, PMAS-Arid Agriculture University, Rawalpindi, Pakistan. After proper examination and on advice of the registered hospital veterinarian, the dogs were euthanized for reasons unrelated to this study. Euthanasia followed the American Veterinary Medical Association (AVMA) guidelines for euthanasia of animals [44]. Briefly, a deep stage of anesthesia was achieved with intramuscular injection of xylazine HCL $(1 \mathrm{mg} / \mathrm{kg})$ and ketamine $(5 \mathrm{mg} / \mathrm{kg})$, in which cardiac arrest was induced by an intravenous injection of supersaturated solution of $\mathrm{MgSO}_{4}(1 \mathrm{~mL} / \mathrm{kg})$. After euthanasia, adipose tissue $(\sim 3-5 \mathrm{~g})$ from the inguinal subcutaneous (SC), omental $(\mathrm{OM})$, and perirenal $(\mathrm{PR})$ region and from the infrapatellar fat pad (IPFP) were isolated as presented in Fig. 1A-D, and kept in Dulbecco phosphate buffer saline (DPBS ${ }^{-1-}$, i.e. without $\mathrm{Ca}^{++}$and $\mathrm{Mg}^{++}$; Sigma-Aldrich, USA) with $5 \%$ penicillin $(100 \mathrm{U} / \mathrm{mL})$, streptomycin $(100 \mu \mathrm{g} / \mathrm{mL})$ and amphotericin-B $(250 \mu \mathrm{g} / \mathrm{mL}$, Caisson, USA) solution and transported to Stem Cell Physiology and Cytogenetic Laboratory at Faculty of Veterinary and Animal Sciences, PMAS-Arid Agriculture University, Rawalpindi, Pakistan. All experimental procedures were performed in strict accordance with the guidelines of the Institutional Animal Ethics Committee.

\section{Cell isolation and culture}

The adipose tissue sample was thoroughly washed with $\mathrm{DPBS}^{-/-}$having 5\% penicillin-streptomycin, amphotericin-B solution, and sliced to prepare a slurry. This suspension was digested with type I collagenase $(0.1 \mathrm{mg} /$ $\mathrm{mL}$; Solarbio, China) in Dulbecco's Modified Eagle's Medium-Low Glucose (DMEM-LG, Bio West; France) at $37^{\circ} \mathrm{C}$ for $135 \mathrm{~min}$. The enzymatic activity of collagenase was stopped by adding an equal amount of LG-DMEM containing $10 \%$ fetal bovine serum (FBS; Bio West, France). The cells released during the digestion process were sieved through a $100 \mu \mathrm{m}$ mesh (Corning, USA) and centrifuged at $548 \mathrm{~g}$ for $10 \mathrm{~min}$ at room temperature. The recovered cell pellet was resuspended in complete LGDMEM (supplemented with 10\% FBS, 1\% penicillinstreptomycin and amphotericin-B) and seeded in T-25 tissue culture flasks. The cultured cells were incubated in a humidified environment at $37^{\circ} \mathrm{C}$ and $5 \% \mathrm{CO}_{2}$. The medium was changed every $48 \mathrm{~h}$ until the cells reach 80 to $90 \%$ confluence. The confluent cells were washed twice with $\mathrm{DPBS}^{-1-}$ and treated with a working solution of trypsin-EDTA $(0.05 \%$ and $0.53 \mathrm{mMw} / \mathrm{v}$, respectively; Caisson, USA) for $10 \mathrm{~min}$ in a humidified environment at $37^{\circ} \mathrm{C}$ with $5 \% \mathrm{CO}_{2}$, trypsin activity was halted with complete LG-DMEM. The recovered cells were centrifuged at $548 \mathrm{~g}$ for $5 \mathrm{~min}$ and sub-cultured till passage number two (P-2).

\section{Mesenchymal stem cells doubling time}

The cell doubling times at P-3 was calculated on day 3, 6,9 , and 12 . Briefly, 5000 cells/well were cultured in a 48-well cell culture plate. On each calculation day, the medium was removed, and the cells were washed twice with $\mathrm{DPBS}^{-1-}$. Subsequently, the cells were trypsinized as described in the previous subsection. Recovered cells were suspended in $1 \mathrm{ml}$ of complete LG-DMEM and counted using a modified Neubauer chamber. Cell viability was assessed by the Trypan blue exclusion test (> 92\%).

\section{Mesenchymal stem cells metabolic assay}

The metabolic activity of MSCs was determined at 3, 6, 9 , and 12 days of culture using the 3-(4,5-dimethylthiazol-2-yl)-2, 5-diphenyltetrazolium bromide (MTT) dye. Briefly, 5000 cells/well were cultured in 48-well culture plates in complete LG-DMEM. The metabolic activity of the cells was evaluated by adding $0.25 \mu \mathrm{g} / \mathrm{ml}$ MTT dye, and incubated for $180 \mathrm{~min}$ at $37^{\circ} \mathrm{C}$ in a humidified chamber containing $5 \% \mathrm{CO}_{2}$. The assay measures the ability 
of cells to reduce MTT into blue formazan crystals with mitochondrial dehydrogenase enzyme. After removing the supernatant, the obtained formazan crystals were mixed into $100 \mu$ l of dimethyl sulfoxide (DMSO; SigmaAldrich, USA), and the absorbance was recorded at $630 \mathrm{~nm}$ using a microplate reader (BioTek 800TS, USA).

\section{Immunophenotyping of mesenchymal stem cells}

Flow cytometric analysis was performed at P3 for quantitative expression of MSC antigens. Briefly, after trypsinization, $5 \times 10^{5}$ cells were pelleted from the culture and resuspended in $\mathrm{DPBS}^{-1-}$. Polyclonal rabbit-raised primary antibodies against CD73 (E-AB-10944), CD90 (E-AB-16098), CD105 (E-AB-34276) (Elab Science, USA), diluted (1:100) in DPBS ${ }^{-1-}$ were added and incubated at $37^{\circ} \mathrm{C}$ for $15 \mathrm{~min}$. The cells were then washed with $\mathrm{DPBS}^{-1-}$ and centrifuged at $500 \mathrm{~g}$ for $5 \mathrm{~min}$ at room temperature to remove unconjugated antibodies. The secondary antibody (anti-IgG) conjugated with Alexa Flour-488 was diluted in DPBS ${ }^{-1-}(1: 300)$ and added for incubation at $37^{\circ} \mathrm{C}$ for $15 \mathrm{~min}$ (separate FACS tubes for each antigen). Afterwards, the cells were washed with DPBS $^{-l-}(300 g)$ and processed for flow cytometry. For quantification of CD45 (ED7018, Exbio, Czech Republic), the mouse raised antibodies coupled with FITC (1:100) were used. The expression of cell surface markers was carried out by flow cytometry (FACScan, BD Biosciences, USA) using CELLQuest (BD Biosciences, USA) software.

In order to visually determine the antigens (CD73, CD90, CD105, FABP4 (E-AB-60028; Elab Science, USA) and osteopontin (ab63856, abcam, UK), the cells were grown on $6 \mathrm{~mm}$ round glass coverslips. After reaching $70 \%$ confluence, the cells were fixed with $4 \%$ buffered formalin for $20 \mathrm{~min}$, washed with $\mathrm{DPBS}^{-/}$, treated with $0.3 \%$ Triton X-100, and blocked with $10 \%$ goat serum. The cells were incubated with polyclonal rabbit-raised primary antibodies against CD73, CD90, CD105, FABP4, and osteopontin (diluted 1:50 in $\mathrm{DPBS}^{-/-}$) for $1 \mathrm{~h}$ at room temperature, and then overnight at $4{ }^{\circ} \mathrm{C}$. Afterwards, the cells were washed with DPBS $^{-1-}$ and incubated with Alexa Flour 594-conjugated secondary antibody (1:400) at room temperature in the dark for $45 \mathrm{~min}$. Next, cells were washed twice with $\mathrm{DPBS}^{-1-}$ and incubated with $0.2 \mu \mathrm{g} / \mathrm{mL}$ of DAPI stain for $5 \mathrm{~min}$ in the dark. Cells were thoroughly washed with $\mathrm{DPBS}^{-1-}$, and mounted to the glass slides with an anti-fading mounting media (Vecta shield, UK). The fluorescence signal was evaluated on an epi-fluorescence microscope (Leica DMI 6000B, 63× objective).

\section{Bi-linage MSCs differentiation assay}

At P3, in vitro differentiation of MSCs was performed into adipocytes and osteocytes. For the adipogenic differentiation assay, $2.5 \times 10^{4}$ cells/well were seeded in 24-well plates for confluence. Cells were provided with an adipogenic induction medium that was LGDMEM supplemented with 10\% FBS, $0.1 \mathrm{mM}$ IBMX (Sigma-Aldrich, USA), $10 \mu \mathrm{M}$ rosiglitazone, $0.3 \mathrm{mM}$ dexamethasone, $5 \mu \mathrm{g} / \mathrm{mL}$ insulin (Nova Nordisk, Denmark), and $1 \%$ penicillin-streptomycin, and amphotericin-B. After $48 \mathrm{~h}$, the adipogenic induction medium was replaced with adipogenic maintenance medium, which composed of LG-DMEM supplemented with $1 \%$ Ex-cyte (Millipore, USA), $5 \mu \mathrm{g} / \mathrm{mL}$ insulin, $1 \%$ penicillin-streptomycin, and amphotericin-B for 7 days with medium change after every $48 \mathrm{~h}$. Before staining with oil-red-O (ORO), the cells were washed with DPBS ${ }^{-/-}$ and fixed with $4 \%$ buffered formalin (Sigma-Aldrich, USA) at room temperature for $30 \mathrm{~min}$ and rinsed twice with $\mathrm{DPBS}^{-1-}$ to remove the traces of formalin. Subsequently, the working solution of ORO (6:4) was poured into all the wells and incubated in the dark at room temperature for $30 \mathrm{~min}$. Cells were rinsed with $\mathrm{DPBS}^{-1-}$ and visualized under an inverted light microscope. The ORO stain was eluted from the cells using anhydrous isopropanol, the absorbance measured at $490 \mathrm{~nm}$ and normalized as follow:

ORO Quantification = ORO Concentration/Cell Number $\times 100$

For osteogenic differentiation, $2.5 \times 10^{4}$ cells/well were seeded in 24-well plates. After reaching confluence, the cells were provided with an osteogenic medium consisting of $\alpha$-MEM supplemented with $10 \%$ FBS, $10 \mathrm{mM} \beta$-glycerophosphate (Sigma-Aldrich, USA), $50 \mu \mathrm{M}$ ascorbate-2-phosphate, $100 \mathrm{nM}$ dexamethasone, $0.75 \mathrm{nM}$ vitamin- $\mathrm{D}_{3}, 1 \%$ penicillin-streptomycin and amphotericin-B. After 21 days of incubation, the cells were stained with Alizarin Red Stain (ARS; Sigma-Aldrich, USA) to assess the degree of mineralization. Briefly, the medium was removed, and the wells were washed twice with $\mathrm{DPBS}^{-1-}$. The cells were fixed with $4 \%$ formalin for $30 \mathrm{~min}$, and washed again with DPBS $^{-1-}$. Then, ARS working solution $(40 \mathrm{mM})$ was added and cells were incubated for $45 \mathrm{~min}$ in the dark. The dye was removed, cells were washed thrice with $\mathrm{DPBS}^{-1-}$ and observed under an inverted light microscope.

\section{Alkaline phosphatase activity (ALP)}

After 21 days of osteogenesis, ALP activity was evaluated using a commercially available kit (ELITech Group, France) according to the manufacturer's instructions. After cell lysis, p-nitrophenyl phosphate (p-NPP) was used as a substrate to detect ALP activity and normalized to the total protein present in the cell lysate detected 
Table 1 The given primer sequences used to amplify specific genes of the canine mesenchymal stem cells and differentiated adipocytes/osteocytes

\begin{tabular}{|c|c|c|c|}
\hline Gene & Sense $5^{\prime}-3^{\prime}$ & Anti-sense $3^{\prime}-5^{\prime}$ & $\begin{array}{l}\text { Amplicon } \\
\text { size (bp) }\end{array}$ \\
\hline CD 73 & TTTGGGGAAACCTTTGACC & AGAGGCTCGTAACTGGGTACTC & 116 \\
\hline CD 90 & CGGCTTCACCACCAAGGACG & TCTGGGCCAGCAGGCTTATG & 140 \\
\hline CD 105 & CCTCAGTGCAAAGAAGAAT & CTTGGAAGATCAGTTTGGGG & 89 \\
\hline FAS & GGCTGGAGCCGGCTACTGCC & ATTCAGGATGGTAGCGTACA & 94 \\
\hline FABP4 & CACCATTAAATCAGAAAGCACC & CCAGGACACCTCCATCTAAG & 128 \\
\hline PPARY & TAAAGAGCCTGAGAAAGCC & GCTTCACATTCAGCAAACC & 156 \\
\hline Osterix & TGCTTGAGGAGGAAGCTCAC & TTTGGGGGCTGAAAGGTCAC & 161 \\
\hline Osteocalcin & TGCAACCTTCGTGTCCAAG & TGGAAGCCAATGTGGTCAG & 171 \\
\hline Osteopontin & TGATTTTCCCACTGACATTCC & TCCATACTCGCACTTTTCAC & 195 \\
\hline GAPDH & AAGAAGGTAGTGAAGCAGG & GCGTCGAAGGTGGAAGAGTGGG & 212 \\
\hline
\end{tabular}

using a commercially available protein assay kit (Bio-Rad, California, USA) following manufacturer instructions.

\section{Gene expression studies}

Gene expression analysis was performed at the Institute of Veterinary Physiology, Freie Universität Berlin, Berlin, Germany. For this purpose, $1 \times 10^{5}$ cells were seeded in a T-25 cell culture flask. After confluence, the cells were differentiated into the adipogenic or osteogenic lineages. For RNA isolation, the cells were scraped from the cell culture flasks, pelleted ( $548 \mathrm{~g}$, for $5 \mathrm{~min}$ ), transferred to RNA-later (Solarbio, China), and preserved at $-20^{\circ} \mathrm{C}$. The total RNA extraction was performed by using the NucleoSpin ${ }^{\circledR}$ RNA kit (Macherey-Nagel GmbH \& Co. KG, Germany) as per manufacturer's instructions and quantified by using a Nanophotometer (Implen ${ }^{\circledR}$, Munich, Germany). Further, the RNA was reverse transcribed using the iScriptc DNA synthesis kit (Bio-Rad, Munich, Germany) according to the manufacturer's instructions. The gene-specific primer sets for CD73 (NT5E), CD90 (THY1), CD105 (ENDOGLIN), fatty acid synthase (FAS), fatty acid binding protein 4 (FABP4), peroxisome proliferator activated receptor gamma $(P P A R-\gamma)$, osteopontin (SPP1), osteocalcin (BGLAP), and osterix (SP7) were used in this study (Table.1). The qPCR reaction was performed in iCycler (Thermo Scientific, Massachusetts, USA) with SYBR green master mix (Bio-Rad, Munich, Germany) and the conditions were: initial denaturation at $94^{\circ} \mathrm{C}$ for $3 \mathrm{~min}$ followed by 40 cycles of denaturation at $94{ }^{\circ} \mathrm{C}$ for $15 \mathrm{~s}$, primer annealing at $58^{\circ} \mathrm{C}$ for $25 \mathrm{~s}$ and primer extension at $72^{\circ} \mathrm{C}$ for $1 \mathrm{~min}$ and with a final hold at $72{ }^{\circ} \mathrm{C}$ for $1 \mathrm{~min}$. All reactions were performed in triplicate while keeping the $G A P D H$ as a housekeeping gene. The $2^{-\Delta \Delta C T}$ method was performed [45] to determine relative expression of target genes where a pool of undifferentiated cells from P3 were used as calibrator.

\section{Statistical analysis}

The data sets presented in this manuscript were statistically analyzed and graphs were plotted by using Sigma Plot 12.0 software (Systat Software Inc., San Jose, CA, USA). The experiment was performed in duplicate with three different animals, each of which served as an experimental unit. The observations of doubling time, ORO quantification, ALP activity, and flow cytometric analysis were arithmetically pooled from 2 wells of 6-well, 24-well, or 48-well cell culture plate where appropriate. The qPCR was conducted in triplicate. The gene expression of $C D 73, C D 90$, and $C D 105$ was analyzed by using one-way ANOVA. At the same time, all other data (doubling time/metabolic assay, ORO quantification, ALP activity, adipogenic, and osteogenic gene expression) were analyzed by two-way ANOVA, in which the interaction was maintained in the form of tissue $x$ individual determinations. The significance among different groups was identified by Holm-Sidak post-hoc test. The data are expressed as mean \pm standard error of the mean (SEM), where $P<0.05$ is considered statistically significant.

\section{Authors' contributions}

M.S., J.A. designed the study. M.S., U.R., E.S. collected the samples and conducted the experiment. S.W. performed the FACS analysis. S.B. and G.S. did gene expression studies. A.Y., M.Y., M.D., G.S., and J.A. analyzed and reviewed the data. U.R., M.S., and A.Y. wrote the manuscript. All authors studied/finalized and approved the manuscript.

\section{Funding}

This study was supported by the Higher Education Commission of Pakistan (NRPU-7830) for experimental and academic work given in the manuscript. Gene expression analysis was carried out at the Freie Universität Berlin supported by funding from the Alexander von Humboldt Foundation Alumni Research Fellowship and an Elsa Neumann stipend from the state of Berlin. 
All funding agencies had no role in study design, interpretation of data and reporting of the study.

\section{Availability of data and materials}

The datasets used and/or analyzed during the current study are available from the corresponding author on reasonable request.

\section{Declarations}

\section{Ethics approval, guidelines, and consent to participate}

All animal care and in vitro experimental procedures were carried out in accordance with the guidelines of the University Ethics Committee of the PMAS-Arid Agriculture University, Rawalpindi complies with the ARRIVE guidelines.

\section{Consent for publication}

All authors have read and agreed to publish the manuscript.

\section{Competing interests}

The authors declare that we don't have any intellectual and financial conflict of interests with a person or institution.

\begin{abstract}
Author details
${ }^{1}$ Department of Clinical Studies, Faculty of Veterinary and Animal Sciences, PMAS-Arid Agriculture University, Rawalpindi 46300, Pakistan. ${ }^{2}$ Department of Veterinary Biomedical Sciences, Faculty of Veterinary and Animal Sciences, PMAS-Arid Agriculture University, Rawalpindi 46300, Pakistan. ${ }^{3}$ Department of Animal Breeding and Genetics, Faculty of Veterinary and Animal Sciences, PMAS-Arid Agriculture University, Rawalpindi 46300, Pakistan. ${ }^{4}$ ABO Scientific, Rawalpindi 46300, Pakistan. ${ }^{5}$ Institute of Veterinary-Physiology, Freie Universität Berlin, Berlin, Germany.
\end{abstract}

\section{Received: 3 June 2021 Accepted: 29 November 2021}

\section{Published online: 18 December 2021}

\section{References}

1. Wei X, Yang X, Han ZP, Qu FF, Shao L, Shi YF. Mesenchymal stem cells: a new trend for cell therapy. Acta Pharmacol Sin. 2013;34(6):747-54.

2. Vieira NM, Brandalise V, Zucconi E, Secco M, Strauss BE, Zatz M. Isolation, characterization, and differentiation potential of canine adipose-derived stem cells. Cell Transplant. 2010;19(3):279-89.

3. Kang BJ, Ryu HH, Park SS, Koyama Y, Kikuchi M, Woo HM, et al. Comparing the osteogenic potential of canine mesenchymal stem cells derived from adipose tissues, bone marrow, umbilical cord blood, and Wharton's jelly for treating bone defects. JVet Sci. 2012;13(3):299-310.

4. Sasaki A, Mizuno M, Ozeki N, Katano H, Otabe K, Tsuji K, et al. Canine mesenchymal stem cells from synovium have a higher chondrogenic potential than those from infrapatellar fat pad, adipose tissue, and bone marrow. PLoS One. 2018;13(8):e0202922.

5. Dominici M, Le Blanc K, Mueller I, Slaper-Cortenbach I, Marini FC, Krause DS, et al. Minimal criteria for defining multipotent mesenchymal stroma cells. The International Society for Cellular Therapy position statement. Cytotherapy. 2006;8(4):315-7.

6. Zhu Y, Liu T, Song K, Fan X, Ma X, Cui Z. Adipose-derived stem cell: a better stem cell than BMSC. Cell Biochem Funct. 2008;26(6):664-75.

7. Hoffman A, Dow S. Concise review: stem cell trials using companion animal disease models. Stem Cells Express. 2016;34(7):1709-29.

8. Kriston-Pál É, Czibula Á, Gyuris Z, Balka G, Seregi A, Sükösd F, et al. Characterization and therapeutic application of canine adipose. Can J Vet Res. 2017;81(1):73-8.

9. Black LL, Gaynor J, Gahring D, Adams C, Aron D, Harman S, et al. Effect of adipose-derived mesenchymal stem and regenerative cells on lameness in dogs with chronic osteoarthritis of the coxofemoral joints: a randomized, double-blinded, multicenter, controlled trial. Vet Ther. 2007;8(4):272-84.

10. Guercio A, Di Marco P, Casella S, Cannella V, Russotto L, Purpari G, et al. Production of canine mesenchymal stem cells from adipose tissue and their application in dogs with chronic osteoarthritis of the humeroradial joints. Cell Biol Int. 2012;36(2):189-94.

11. Neupane M, Chang CC, Kiupel M, Yuzbasiyan-Gurkan V. Isolation and characterization of canine adipose-derived mesenchymal stem cells. Tissue Eng - Part A. 2008;14(6):1007-15.

12. Bahamondes F, Flores E, Cattaneo G, Bruna F, Conget P. Omental adipose tissue is a more suitable source of canine mesenchymal stem cells. BMC Vet Res. 2017;13(1):1-9.

13. Guercio A, Di Bella S, Casella S, Di Marco P, Russo C, Piccione G. Canine mesenchymal stemcells (MSCs): characterization in relation to donor age and adipose tissue-harvesting site. Cell Biol Int. 2013;37(8):789-98.

14. Hoogduijn MJ, Crop MJ, Peeters AMA, Van Osch GJVM, Balk AHMM, Ijzermans JNM, et al. Human heart, spleen, and perirenal fat-derived mesenchymal stem cells have immunomodulatory capacities. Stem Cells Dev. 2007;16(4):597-604.

15. Reber S, Goehring NW. Intracellular scaling mechanisms. Cold Spring Harb Perspect Biol. 2015;7(12):a019067.

16. Kitami T, Logan DJ, Negri J, Hasaka T, Tolliday NJ, Carpenter AE, et al. A chemical screen probing the relationship between mitochondrial content and cell size. PLoS One. 2012;7(3):e33755.

17. Sandhu MA, Jurek S, Trappe S, Kolisek M, Sponder G, Aschenbach JR. Influence of bovine serum lipids and fetal bovine serum on the expression of cell surface markers in cultured bovine preadipocytes. Cells Tissues Organs. 2017:204(1):13-24.

18. Zuk PA, Zhu M, Ashjian P, De Ugarte DA, Huang Jl, Mizuno H, et al. Human adipose tissue is a source of multipotent stem cells. Mol Biol Cell. 2002:13:4279-95.

19. Gao ZW, Dong K, Zhang HZ. The roles of CD73 in cancer. Biomed Res Int. 2014:2014:460654.

20. Requicha JF, Viegas CA, Albuquerque CM, Azevedo JM, Reis RL, Gomes ME. Effect of anatomical origin and cell passage number on the stemness and osteogenic differentiation potential of canine adipose-derived stem cells. Stem Cell Rev Reports. 2012;8(4):1211-22.

21. Kumar A, Bhanja A, Bhattacharyya J, Jaganathan BG. Multiple roles of CD90 in cancer. Tumor Biol. 2016:37(9):11611-22.

22. Sakaguchi Y, Sekiya I, Yagishita K, Muneta T. Comparison of human stem cells derived from various mesenchymal tissues: superiority of synovium as a cell source. Arthritis Rheum. 2005:52(8):2521-9.

23. Takemitsu H, Zhao D, Yamamoto I, Harada Y, Michishita M, Arai T. Comparison of bone marrow and adipose tissue-derived canine mesenchymal stem cells. BMC Vet Res. 2012;8:150.

24. Nadri S, Soleimani M, Hosseni RH, Massumi M, Atashi A, Izadpanah R. An efficient method for isolation of murine bone marrow mesenchymal stem cells. Int J Dev Biol. 2007;51(8):723-9.

25. Anderson P, Carrillo-Gálvez AB, García-Pérez A, Cobo M, Martín F. CD105 (Endoglin)-negative murine mesenchymal stromal cells define a new multipotent subpopulation with distinct differentiation and immunomodulatory capacities. PLoS One. 2013;8(10):e76979.

26. De Bakker E, Van Ryssen B, De Schauwer C, Meyer E. Canine mesenchymal stem cells: state of the art, perspectives as therapy for dogs and as a model for man. Vet Q. 2013;33(4):225-33.

27. Xu S, De Becker A, Van Camp B, Vanderkerken K, Van Riet I. An improved harvest and in vitro expansion protocol for murine bone marrow-derived mesenchymal stem cells. J Biomed Biotechnol. 2010;2010:105940.

28. Sekiya I, Larson BL, Vuoristo JT, Cui JG, Prockop DJ. Adipogenic differentiation of human adult stem cells from bone marrow stroma (MSCs). J Bone Miner Res. 2004;19(2):256-64.

29. Ritter A, Friemel A, Roth S, Kreis NN, Hoock SC, Safdar BK, et al. Subcutaneous and visceral adipose-derived mesenchymal stem cells: commonality and diversity. Cells. 2019:8(10):1-23.

30. Gurriarán-Rodríguez U, Al-Massadi O, Roca-Rivada A, Crujeiras AB, Gallego $\mathrm{R}$, Pardo $\mathrm{M}$, et al. Obestatin as a regulator of adipocyte metabolism and adipogenesis. J Cell Mol Med. 2011;15(9):1927-40.

31. Aldridge A, Kouroupis D, Churchman S, English A, Ingham E, Jones E. Assay validation for the assessment of adipogenesis of multipotential stromal cells-a direct comparison of four different methods. Cytotherapy. 2013;15(1):89-101.

32. Zhang JS, Lei JP, Wei GQ, Chen H, Ma CY, Jiang HZ. Natural fatty acid synthase inhibitors as potent therapeutic agents for cancers: a review. Pharm Biol. 2016;54(9):1919-25. 
33. Jang WG, Kim EJ, Kim DK, Ryoo HM, Lee KB, Kim SH, et al. BMP2 protein regulates osteocalcin expression via Runx2-mediated Atf6 gene transcription. J Biol Chem. 2012;287(2):905-15.

34. Reddi S, Shanmugam VP, Tanedjeu KS, Kapila S, Kapila R. Effect of buffalo casein-derived novel bioactive peptides on osteoblast differentiation. Eur J Nutr. 2018;57(2):593-605.

35. Liu F, Akiyama Y, Tai S, Maruyama K, Kawaguchi Y, Muramatsu K, et al. Changes in the expression of CD106, osteogenic genes, and transcription factors involved in the osteogenic differentiation of human bone marrow mesenchymal stem cells. J Bone Miner Metab. 2008;26(4):312-20.

36. Nakashima K, Zhou X, Kunkel G, Zhang Z, Deng JM, Behringer RR, et al. The novel zinc finger-containing transcription factor osterix is required for osteoblast differentiation and bone formation. Cell. 2002;108(1):17-29.

37. Langenbach F, Handschel F. Effects of dexamethasone, ascorbic acid and $\beta$-glycerophosphate on the osteogenic differentiation of stem cells in vitro. Stem Cell Res Ther. 2013;4(5):117.

38. Mikami Y, Omoteyama K, Kato S, Takagi M. Inductive effects of dexamethasone on the mineralization and the osteoblastic gene expressions in mature osteoblast-like ROS17/2.8 cells. Biochem Biophys Res Commun. 2007:362(2):368-73.

39. Sanghani-Kerai A., Black C, Cheng S. O, Collins L, Schneider, N, Blunn G, et al. Clinical outcomes following intra-articular injection of autologous adipose-derived mesenchymal stem cells for the treatment of osteoarthritis in dogs characterized by weight-bearing asymmetry. Bone Joint Res 2021;10(10):650-658.

40. Rashid U, Sandhu MA, Yaqoob M, Yousaf A. Critical bone gap repair using autologous adipose derived canine mesenchymal stem cell graft. Pak Vet J. 2021;41(4):513-18.

41. Aksu AE, Rubin JP, Dudas JR, Marra KG. Role of gender and anatomical region on induction of osteogenic differentiation of human adiposederived stem cells. Ann Plast Surg. 2008:60(3):306-22.

42. Martinello T, Bronzini I, Maccatrozzo L, Mollo A, Sampaolesi M, Mascarello F, et al. Canine adipose-derived-mesenchymal stem cells do not lose stem features after a long-term cryopreservation. Res Vet Sci. 2011;91(1):18-24.

43. Prządka P, Buczak K, Frejlich E, Gąsior L, Suliga K, Kiełbowicz Z. The role of mesenchymal stem cells (MSCs) in veterinary medicine and their use in musculoskeletal disorders. Biomolecules. 2021;11(8):1141.

44. AVMA (American Veterinary Medical Association). AVMA Guidelines for the Euthanasia of Animals: 2020 Edition. 2020, AMVA, Schaumburg, Illinois.

45. Livak KJ, Schmittgen TD. Analysis of relative gene expression data using real-time quantitative PCR and the 2- $\triangle \triangle C T$ method. Methods. 2001;25(4):402-8.

\section{Publisher's Note}

Springer Nature remains neutral with regard to jurisdictional claims in published maps and institutional affiliations.

Ready to submit your research? Choose BMC and benefit from:

- fast, convenient online submission

- thorough peer review by experienced researchers in your field

- rapid publication on acceptance

- support for research data, including large and complex data types

- gold Open Access which fosters wider collaboration and increased citations

- maximum visibility for your research: over $100 \mathrm{M}$ website views per year

At BMC, research is always in progress.

Learn more biomedcentral.com/submissions 